\title{
POWER OF THE PEOPLE: A CASE STUDY USING FACEBOOK AS A DATA SOURCE IN QUALITATIVE RESEARCH
}

\author{
Dr. G. Chris Nelson, Riverside Research, cnelson@riversideresearch.org
}

\begin{abstract}
Social Media Sites (SMS), such as Facebook, have become platforms of communication or virtual "soapboxes" for the expression of ideas. In 2011, Facebook transcended its origins as a social communications platform, and became a communications catalyst that gave voice to political minority. For Egypt, in 2011, this new kind of socialmedia-site-activism erupted into revolution. Accordingly, because of the part it played in the events surrounding the Egyptian revolution, Facebook has become a vogue source of data for scholarly research. This paper examines the Facebook activities captured during the Egyptian revolution as a case study. This paper strives to provide a critical look at the advantages and limitations, of utilizing the largely unstructured data contained in Facebook, for qualitative academic research. The Researcher posits that while social media and Facebook are unique data sets, there is still a considerable amount of methodological and protocol work to be accomplished before they can be successfully integrated as a widely utilized data source in qualitative research.
\end{abstract}

Keywords: Social Media, Facebook, Egyptian Revolution, Qualitative, Case Study, Meta-Analysis

\section{INTRODUCTION}

Social Media Sites, such as Facebook, have become platforms of communication or virtual "soapboxes" for the expression of ideas, and are utilized by a large portion of the world's population. Facebook has ingrained itself into the $21^{\text {st }}$ century consciences as a new overarching kind of social interaction - a unique cultural matrix. A key distinction, between SMSs and traditional communications technologies, is that this new cultural matrix permeates our other cultural matrices since it is always available. Facebook unites disparate aspects of our lives, connecting our traditional cultural matrices in new ways. In 2011, Facebook transcended its origins as a simple communication platform, and became a communications catalyst that gave voice to political minority. For Egypt, in 2011, this new type of Social Media Site-driven activism erupted into revolution. Accordingly, because of the part it played in the events surrounding the Egyptian revolution Facebook is becoming a vogue source of data, for scholarly research. This paper aims to provide a critical look at the advantages and limitations, of utilizing the largely unstructured data contained in Facebook, for qualitative research.

\section{Digital Self \& Facebook}

Facebook was founded in a Harvard dorm room in 2004 by Mark Zuckerberg and over the past ten years has become the dominant SMS on the Internet. While much has been made of its genesis (including an Oscar winning film "the social network") the truly important thing to note, for this paper, about Facebook's rise to dominance is that it utilized a construct "which mimicked offline social interaction in the online world" [20]. This is unique in the world of SMSs and the study of "digital self". Until the rise of Facebook the idea of a digital persona, or self, projected into digital arenas was largely considered to be less "rich", and while providing useful data, inherently limited. [13]. As early as 1995, when the Internet as we know it was coalescing, scholars like Hugh Miller were discussing the ideas of presenting oneself in digital arenas. "Electronic communication (EC) has established a new range of frames of interaction with a developing etiquette. Although apparently more limited and less rich than interactions in which the participants are physically present, it also provides new problems and new opportunities in the presentation of self [22]." Other scholars, like Christine Hine were developing methodological models, with sociological roots, to determine cultures, and identities of online denizens [13]. Interestingly, problems of self, like social isolation, appeared online, and seemed to exacerbate real life isolation [21]. Although a relatively new field of study, there is a wealth of literature discussing online identity and community [9]. While initial scholarship suggested that these online personas were mere shades of our true selves, or outright falsehoods, with " less rich" interactions they continued to evolve. As technology developed, the experience improved. Millennial youth, raised 
with a digital presence, have started to use social media, online gaming and other forms of electronic communications as part of their social network, or unique cultural matrices [17, 18, 23, 25]. Subsequently, individuals who made up groups, like the "we are all Khaled Said" Facebook page during the 2011 Egyptian revolution, developed almost completely online. These groups seem to have their own identities and dynamics [12].

\section{We Are All Khaled Said}

Social media, particularly Facebook, was a preferred communications medium for organizing the Egyptian protests, and eventually became a powerful communications tool for opposition event coordination and galvanization of the populace's will [14]. During Egypt's revolution the Facebook page "we are all Khaled Said", in particular, was a bastion for Egyptians who were disaffected by the Mubarak regime. In January 2011, Google executive Wael Ghonim forever changed the course of Egypt's history, and showed the world the potential of social media, by calling for demonstrations on the $25^{\text {th }}$ or National Police day. A native-expatriate-Egyptian, who started the page "we are all Khaled Said", as a way to add his voice to the growing tide of anti-government rhetoric and unleashed a tsunami of vitriol that swept into Tahir Square that fateful morning. Using the "handle" El Shaheed, or "the martyr," he anonymously administered, the "we are all Khaled Said" Facebook page, which over the course of six months had become a beacon for other likeminded protesters [11, 15]. After getting arrested, tortured, and subsequently released, as the regime disintegrated, Ghonim became a global sensation, celebrated by demonstrators, newscasters, academics and politicians from Syria to Hollywood. Accordingly, because of his central actions during the events that unfolded, and because those events occurred on his virtual soapbox, it became very vogue to discuss social media's role in the revolt. The same newscasters, academics and politicians have all deliberated a myriad of issues pertaining to the use of social media in Egypt's revolution, and the Arab spring in general [2, 5, 6,]. Yet, while much has been written about the use of social media, in the Egyptian revolt associated with Arab spring movements of 2011-2012 [2, 19, 24], there are several issues surrounding the data that can be obtained from Facebook for use in qualitative research.

\section{RESEARCH METHODOLOGY}

Since the events that transpired during the Egyptian revolution were shared amongst a large population, during a very specific timeframe, they offer the research community a unique opportunity to "explore a real-life contemporary bounded system" [7]. The bounded nature of the events, confined to the Facebook page "we are all Khaled Said", during the Egyptian Revolution and specifically relating to the fall of the Mubarak regime, offers an example that can be utilized for generalization, and as a basis for the discovery of issues surrounding the datasets that can be gleaned for academic endeavors. The value of such case study research is that it allows the investigation of Facebook as a data source, in a practical real-life situation. Therefore, for scholars striving to make sense of the unstructured data in Facebook, and potentially other SMSs, the advantages of a case study approach could be significant. This paper will present a detailed, systematic comparison of various themes discovered during a qualitative case study of the Facebook group we are all Khaled Said.

Procedurally, unlike traditional qualitative case studies, the "participants" and "artifacts" are one in the same digital records, confined to a single web based application, open to public members. Therefore, this paper borrowed heavily from qualitative meta-analysis protocols. This additional methodological step allowed the author to clarify concepts and patterns observed by leveraging "the existing states of knowledge" regarding the use of social media sites as qualitative data sets. Additionally, by surveying the literature and applying the current state of "the known" to the data mined from Facebook, it helped us to develop new, or confirm existing, themes and more importantly temper our results [7]. Data collection was accomplished using the QSR incorporated NVivo application. Specifically, data was mined with the NCapture plugin for the Internet Explorer and Chrome web browsers directly from the Facebook group page.

\section{RESULTS}

\section{A Snapshot in Time}

One of the most striking features of Facebook data is its uniqueness. Arguably, encapsulated on the Facebook group page "we are all Khaled Said" is the entirety of the Egyptian revolutionary experience. It is a treasure trove of 
artifacts including pictures, audio, video and contextual sentiments laid out neatly, on a reverse timeline. This unique repository of data freed the researcher from the tyranny of distance and time that removed him from the event, the participant's haze of memory when researching said historical events, and the oftentimes-onerous task of data and artifact collection during periods of extreme and continuing social upheaval. The second thing that was apparent to the researcher was its availability. Since the data was already in a native digital format it was seemingly easy to collect. Which of course meant that (again seemingly) the data was, ready for analysis, barring some minor transformation. Ease of collection and analysis issues will be discussed later in this paper, however, the essential assessment of Facebook's uniqueness as a data source cannot be understated. Facebook offers an enormous record of qualitative data related to emerging, ongoing and historical events - as they happened. As has been noted by Grant Blank, and other scholars, Facebook (and other social media sites) represent a revolutionary turn for qualitative research. Potentially, Facebook embodies a potential paradigm shift for the qualitative academic community. Not only does Facebook represent an ability to gain access where it would have been nearly impossible before, but it could also address the problem of contact with large sample populations. Finally, Facebook and potentially other SMSs improve reliability and simplify certain penetration issues. Digital access means that researchers could now get data on entire populations, without issues of biased respondent selection or biased interviewer outcomes [4].

\section{Validity and Continued Validity}

One of the greatest limitations when using Facebook or any other social media site for data collection is the ability to adequately judge the information's validity. Specifically, other research has shown that like-minded individuals tend to congregate together and reinforce existing social networks and attitudes online. This is reminiscent of traditional social network studies, which showed that when people occupied similar network positions, they formed common attitudes. While Facebook and other SMSs have millions of users, the individual experience tends to be homogenized as a result of Facebook's structure and the way user-provided-content strengthens ties. $[1,10,16]$ In the case of the "we are all Khalid Said" Facebook group less than one percent of the artifacts between 10 June 2010 and 14 February 2011 could be construed as remotely pro-regime. While certainly the Mubarak government was unpopular, it is doubtful that less than one percent of the population supported the regime. This would suggest that a sample of the entire population, regardless the Facebook sample size, is impossible to achieve. Therefore, most samples would be convenience samples. The fact that user-provided-content controls the ties that define an individuals' Facebook network could have repercussions for the understanding of behaviors observed within its confines.

Additionally, while mobile web and smartphone technologies have made Facebook and other SMSs more accessible, there will always be a segment of the population that does not utilize them [4]. This is especially true in less technologically developed nations, like Egypt. Historical accounts back up the data the researcher collected in that expatriates, the intelligencia, and urban youth were the primary members of the "we are all Khaled Said" Facebook group, and not the conservative, impoverished, or tribal peoples - who make up the majority of Egyptian society. Unfortunately, there was no way to accurately discern the delta between the user population, mobile technology subscribers, and Facebook members. This could be especially true in developing nations, or authoritian regimes where data points on digital population segments are unavailable at best, or nonexistent at worst. Therefore, data collected must be tempered by additional contextual framing. In this case study, historical records generated by traditional media sources were employed to aid in understanding during coding. This additional data gave context to the historical events on Facebook as they unfolded. Unfortunately, the researcher did not discover any models to aid in understanding of events that are transpiring while in real time. It seems that only in retrospect, or with some supplemental data sources, will the true scope of behaviors occurring on Facebook and other SMSs be truly discernable.

Finally, specifically with Facebook, group pages can continue to have activity, beyond the timeframe being studied. This is a double-edged sword since researchers need to have access to the group page (so it must remain active) but they simultaneously also want it to stay static, and preserve the experience as it happened. Researchers have no way of knowing whether or not the pages are continually being edited for content, if minority comments or artifacts are being removed or if history (on the page) is literally being rewritten. In the case of "we are all Khaled Said" Facebook page there were a three posts, that were referenced in Wael Ghonim's book [11], which did not exist on the Facebook Group Page. Fortunately, they were so few that they did not really effect the results, however, it did raise a concern about the continuing digital efficacy of social media datasets. The experience also reinforced to us 
that data must be collected as close to the event, as possible, to retain the most accurate record. Of course, for researchers who set up a purpose built Facebook group to accomplish a specific research goal this is not a concern, since they are the administrator of the group. However, for those utilizing Facebook as a data source to understand historical events, it is a real and valid issue.

\section{Ethical Considerations}

Facebook, in many ways, turns ethical concerns for qualitative research, of human subjects, on its head. Empirically, if researchers are looking at historical Facebook pages, or existing groups as nonparticipant observers then there is no human interaction, as it is traditionally defined in the social sciences. However, an inordinate amount of personal data is available to the researcher via Facebook because of user-provided content. When coupled with the aid of a search engine, like Google, researchers potentially have access to a vast amount of personal information without informed consent or the research ever being reviewed by an Institutional Review Board. Additionally, after publication, it could be relatively easy for readers to discern identities and personal facts based on conceptual statements or examples contained in the research, because of the unwitting participants continued digital footprint on Facebook [4]. These concerns are exacerbated when tumultuous events are ongoing, and have truly life or death consequences - like those of the Egyptian revolution. Traditionally in the social sciences, ethical considerations are often based on privacy and informed consent. However, in the new social media paradigm privacy is a thin veneer for most of the digitally active. Unfortunately, the need for ethical adherence and protection of the participants, especially in volatile environments, could introduce a level of researcher bias. This was especially true for this case study, where specific examples could have given deeper meaning to the generalizations made about the targeted Egyptian population, but would have put the persons associated to those examples at risk from the current regime. Finally, another consideration discovered was that depending on the privacy settings of the Facebook group page, identifying data can potentially be obtained and triangulated from an outside search engine. Readers of the published work can utilize the information contained therein to potentially identify individual participants without even being a member of the group, or having a Facebook account. This further complicates the ethical considerations for the researcher when utilizing Facebook as a data source.

\section{Not All Unicode Is Equal}

When conducting qualitative research, the researcher is the main tool for analysis [7, 8]. However, due to the large volumes of data that social media sites like Facebook offer to researchers automated analytical tools are necessary. Programs, like NVivo, atlas ti and Dedoose, can help researchers with their analyses, specifically coding. When coupled with data analysis software like Data Miner, SPSS, and SAS, researchers have a formidable arsenal of computer-based assistance for large data sets. For this case study, NVivo was selected, based on two distinct factors - Unicode support and the NVivo NCapture add on.

Data collection was envisioned to be fairly straightforward (see figure 1). After gaining admittance to the "we are all Khaled Said" Facebook group, the datasets were to be automatically harvested using the NCapture plug-in for the Google chrome web browser. By using the Google Chrome browser, with the Google Translate API, a rough English translation was to be simultaneously collected to aid in initial coding. 


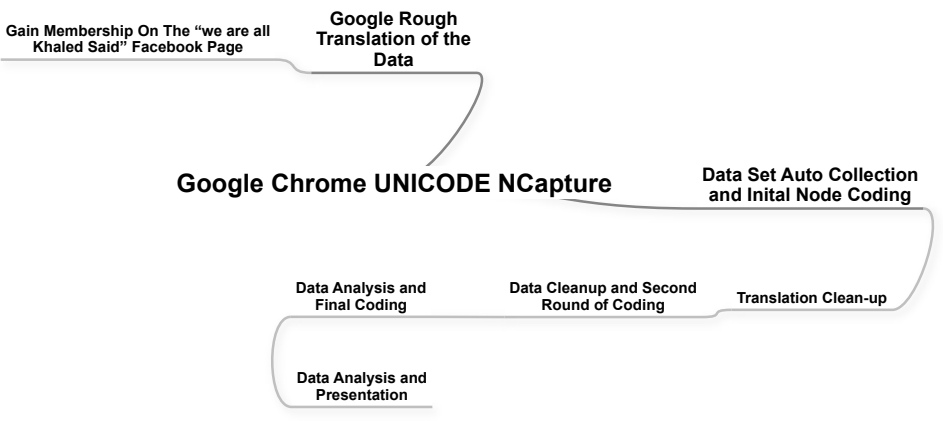

Figure 1. Initial Data Collection Strategy

NVivo Unicode, support was critical because the "we are all Khaled Said" is in Egyptian Arabic. The researcher employed the services of a native Arabic speaker/translator and the final product was to be in English. However, Unicode support for auto translation and rough initial coding (setting nodes upon dataset generation in NVivo) was key to meet the research timeline - based on the quantity of data. Following the dataset generation, and initial auto coding. The concluding trans-literal translation was to be accomplished, inside the NVivo application to give the researcher a single unified dataset for axial coding. Finally, qualitative data analysis, quantitative statistical exports (for use in applications like SPSS) and thematic exploration would all take place inside the NVivo application, giving the researcher a streamlined dataset inside of a common graphical user interface.

Despite the "we are all Khalid Said" data's native digital form and NVivo's capabilities to help the researcher organize, it soon became apparent that data collection and analysis would be incredibly labor intensive (see figure 2). Unfortunately, while NVivo, Google Chrome, and Facebook all support Unicode, they were unable to communicate with each other. NVivo was unable to auto generate a dataset even when the page was largely translated to English, and the researcher had to settle for capturing the data as several, separate (due to size and the Facebook timeline accordion function) Optically Character Recognized (OCR) Portable Document Files (PDFs). Additionally, Chrome, NVivo and Excel (where the researcher resorted to constructing the dataset for importation into NVivo) all had trouble with Unicode support for cut and paste function from right-to-left (Arabic) to left-toright (English).

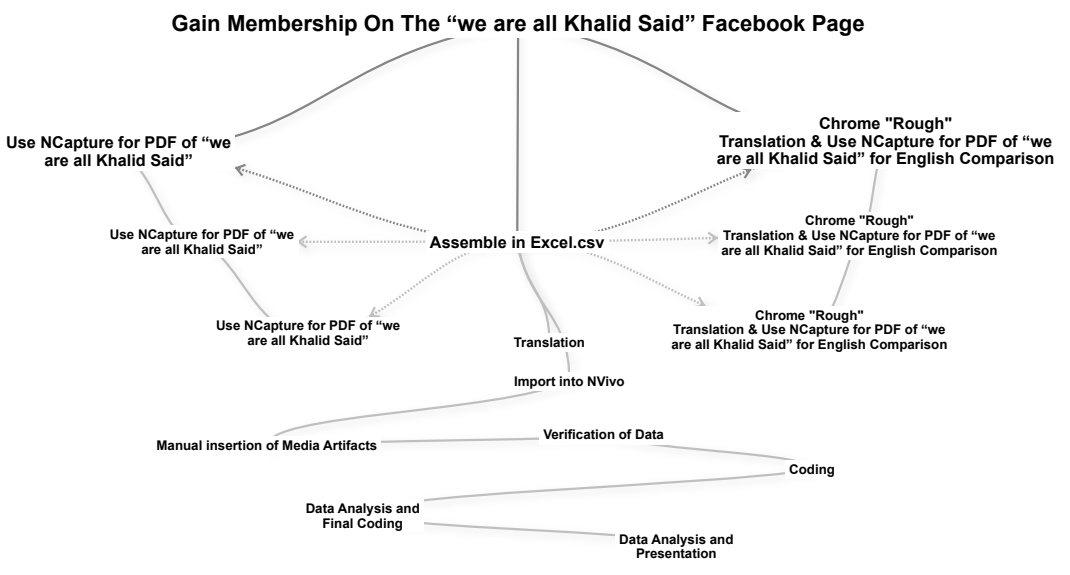

Figure 2. Actual Data Collection

Despite the tribulations of the researcher during the data collection of the "we are all Khalid Said" Facebook Group this is not meant to be a criticism of the software tools available. It does, however, underscore an important point about the utilization of Facebook as a data source, which is: Verification of the dataset is crucial. While this 
researcher's timeline was blown, the he was ultimately was grateful for the digital woes, because it forced him to verify the data, line-by-line, entry-by-entry. Even thought this should be the norm of good scholarship, with Facebook datasets being so large it would have tempting to verify just a portion, and if no errors were found assume that the rest was correct.

\section{CONCLUSIONS}

Social Media Sites and Facebook represent the new digital norm for many people in 2015. With more and more aspects of individuals' lives being catalogued and archived, by SMS sites, it will become an ever-increasing and important warehouse of historical and experiential data for qualitative research. Accessibility and quantity make sites like Facebook unique and invaluable repositories especially as a "new" media outlet. However, unless the analysis is tempered with additional sources the qualitative context could be skewed, due to the user defined experience or engineered hegemony of social media. Additionally, SMS and Facebook represent new ethical hurdles for the qualitative research community. Intuitional Review Boards must deal with the new digital reality and revise their stances on informed consent, or reconcile the loss of some privacy in the $21^{\text {st }}$ century. Finally, like all "big data" datasets Facebook data is a perfect example of "bigger not necessarily being better". Without very structured verification and validity protocols qualitative research could fall victim to skewed or erroneous results. Although social media and Facebook are (rightly so) very vogue in the academic community, there is still a considerable amount of methodological and protocol work to be accomplished before there can be a successful integration as a widely utilized data source in qualitative research.

\section{REFERENCES}

1. Alan Branthwaite, \& Simon Patterson. (2011). The power of qualitative research in the era of social media. Qualitative Market Research: An International Journal, 14(4), 430.

2. Alterman, J. B. (2011). The Revolution Will Not Be Tweeted. The Washington Quarterly, 34(4).

3. Attia, A. M., Aziz, N., Friedman, B., \& Elhusseiny, M. F. (2011). Commentary: The impact of social networking tools on political change in Egypt's "Revolution 2.0." Electronic Commerce Research and Applications, 10(4), 369-374.

4. Blank, G. (2013). Blurring the boundaries: New Social Media, New Social Science (NSMNSS). In International Journal of Market Research (Vol. 55, pp. 461-464). Warc LTD.

5. Bruns, A., Highfield, T., \& Burgess, J. (2013). The Arab Spring and Social Media Audiences English and Arabic Twitter Users and Their Networks. American Behavioral Scientist, 57(7), 871-898.

6. Choudhary, A., Hendrix, W., Lee, K., Palsetia, D., \& Liao, W.-K. (2012). Social media evolution of the Egyptian revolution. Communications of the ACM, 55(5), 74-80.

7. Creswell, J. W. (2003). Research design: qualitative, quantitative, and mixed method approaches. Thousand Oaks, Calif:: Sage Publications.

8. Denzin, N. K., \& Lincoln, Y. S. (2005). The SAGE handbook of qualitative research. Thousand Oaks: Sage Publications.

9. Ellison, N. B. (2007). Social network sites: Definition, history, and scholarship. Journal of Computer-Mediated Communication, 13(1), 210-230.

10. Ellison, N. B., \& Boyd, D. M. (2013). Sociality Through Social Network Sites. The Oxford Handbook of Internet Studies, 151.

11. Ghonim, W. (2012). Revolution 2.0: the power of the people is greater than the people in power : a memoir. Boston: Houghton Mifflin Harcourt.

12. Goggins, S. P., Laffey, J., \& Gallagher, M. (2011). Completely online group formation and development: small groups as socio-technical systems. Information Technology \& People, 24(2), 104-133.

13. Hine, C. (2008). Virtual Ethnography: Modes, Varieties, Affordances. In The SAGE handbook of online research methods (pp. 257-270). SAGE Publications.

14. Hounshell, B. (2011). The Revolution Will Be Tweeted. Foreign Policy, (187), 20-21.

15. Kamal, M., \& Meenalochani, P. (2012). Byte Is Mightier Than The Sword: Egypt Revolution Of 2011. International Journal of Management \& Information Systems (Online), 16(4), 341.

16. 1Kane, G. C., Alavi, M., Labianca, G. (Joe), \& Borgatti, S. P. (2014). What's Different About Social Media Networks? A Framework and Research Agenda. MIS Quarterly, 38(1), 275-304. 
17. Kassam, A. (2013). Changing society using new technologies: Youth participation in the social media revolution and its implications for the development of democracy in sub-Saharan Africa. Education and Information Technologies, 18(2), 253-263.

18. Kelm, O. R. (2011). Social Media It's What Students Do. Business Communication Quarterly, 74(4), 505-520.

19. Lotan, G., Graeff, E., Ananny, M., Gaffney, D., Pearce, I., \& Boyd, D. (2011). The Revolutions Were Tweeted: Information Flows During the 2011 Tunisian and Egyptian Revolutions. International Journal of Communication, 5.

20. McGirt, E. (2007). HACKER. DROPOUT. CEO. (cover story). Fast Company, (115), 74-112.

21. McPherson, M., Smith-Lovin, L., \& Brashears, M. E. (2006). Social isolation in America: Changes in core discussion networks over two decades. American Sociological Review, 71(3), 353-375.

22. Miller, H. (1995). The Presentation of Self in Electronic Life: Goffman on the Internet. Sciences New York, (June).

23. Salimkhan, G., Manago, A. M., \& Greenfield, P. M. (2010). The Construction of the Virtual Self on MySpace. Cyberpsychology, 4(1), 1-18.

24. Sayed, N. (2012). Towards the Egyptian Revolution: Activists perceptions of social media for mobilization. Journal of Arab \& Muslim Media Research, 4(2-3), 2-3.

25. Steinfield, C., Ellison, N., \& Lampe, C. (2008). Net Worth: Facebook Use and Changes in Social Capital Over Time. Conference Papers -- International Communication Association, 1-23. 